\title{
A importância nutricional da merenda escolar para a comunidade
}

\author{
A nutritional importance of school meals for the community \\ Una importancia nutricional de las comidas escolares para la comunidade
}

Recebido: 15/10/2021 | Revisado: 22/10/2021 | Aceito: 25/10/2021 | Publicado: 27/10/2021

\author{
Rosa Gladys Casilla Arqque \\ ORCID: https://orcid.org/0000-0002-5925-0827 \\ Centro Universitário Fametro, Brasil \\ E-mail: gladys30.rosa@gmail.com \\ José Carlos de Sales Ferreira \\ ORCID: https://orcid.org/0000-0002-1867-8229 \\ Centro Universitário Fametro, Brasil \\ E-mail: jcarlos.sales@gmail.com \\ Rebeca Sakamoto Figueiredo \\ ORCID: https://orcid.org/0000-0002-9819-8099 \\ Centro Universitário Fametro, Brasil \\ E-mail: rebeca.figueiredo@fametro.edu.br
}

\begin{abstract}
Resumo
Introdução: A merenda escolar ou simplesmente merenda refere-se à refeição que os estudantes têm dentro das escolas, especialmente durante os intervalos, e está instituída nas escolas públicas do Brasil pelo PNAE, criado em meados da década de 1950. Objetivo geral: analisar a importância da merenda escolar para os estudantes. Metodologia: $\mathrm{O}$ estudo foi realizado com coleta de dados a partir de fontes secundarias, por meio de levantamento bibliográfico e baseado na experiência vivenciada por autores. Resultados e Discussão: a merenda, além de alimentar e nutrir as crianças, também proporciona interação social entre colegas da escola e as cozinheiras. Os professores estimulam o desenvolvimento de bons hábitos alimentares e ensinam como é o sentar-se à mesa e comer adequadamente. Considerações Finais: A merenda escolar oferecida nas escolas pública é importante ao desenvolvimento psicofísico do aluno, auxiliando-o em todos os aspectos: físico motor, intelectual, afetivo emocional, econômico e social. Esses aspectos de bem-estar contribuem para que o sujeito tenha condições satisfatórias para aprender.
\end{abstract}

Palavras-chave: Alimentação escolar; Indicadores de saúde comunitária; Alimentos; Dieta e nutrição.

\begin{abstract}
Introduction: The school lunch or simply lunch refers to the food that students have inside schools, especially during breaks, and is instituted in public schools in Brazil by the PNAE, created in a series from the 1950s. General objective: analyze importance of school meals for students. Methodology: The study was carried out with data collection from secondary sources, through a bibliographic survey and based on the authors' experience. Results and Discussion: the lunch, in addition to feeding and nourishing the children, also offers social interaction between schoolmates and the cooks. Teachers encourage the development of good eating habits and teach what it is like to sit at the table and eat in common. Final Considerations: The school lunch offered in public schools is important to the psychophysical development of the student, helping him in all aspects: physical motor, intellectual, emotional affective, economic and social. These aspects of well-being contribute to the subject having satisfactory conditions to learn.
\end{abstract}

Keywords: School meals; Community health indicators; Foods; Diet and nutrition.

\section{Resumen}

Introducción: El almuerzo escolar o simplemente almuerzo se refiere a la comida que los estudiantes tienen dentro de las escuelas, especialmente durante los descansos, y es instituido en las escuelas públicas de Brasil por el PNAE, creado en una serie de la década de 1950. Objetivo general: analizar la importancia de las comidas escolares para estudiantes. Metodología: El estudio se realizó con recolección de datos de fuentes secundarias, a través de un relevamiento bibliográfico y en base a la experiencia de los autores. Resultados y Discusión: el almuerzo, además de alimentar y nutrir a los niños, también ofrece interacción social entre compañeros y cocineros. Los profesores fomentan el desarrollo de buenos hábitos alimentarios y enseñan cómo es sentarse a la mesa y comer en común. Consideraciones finales: El almuerzo escolar que se ofrece en las escuelas públicas es importante para el desarrollo psicofísico del alumno, ayudándolo en todos los aspectos: físico motor, intelectual, emocional afectivo, económico y social. Estos aspectos del bienestar contribuyen a que el sujeto tenga condiciones satisfactorias para aprender.

Palabras clave: Comidas del colegio; Indicadores de salud comunitaria; Alimentos; Dieta y nutrición. 


\section{Introdução}

A merenda escolar ou simplesmente merenda refere-se à refeição que os estudantes têm dentro das escolas, especialmente durante os intervalos. Muitas escolas oferecem aos alunos alimentos preparados na própria instituição, enquanto outras apenas oferecem espaços para que os alunos comam os alimentos trazidos em suas próprias merendeiras ou, como são mais comumente chamadas hoje, lancheiras (Fonseca, 2015).

A merenda escolar está instituída nas escolas públicas do Brasil pelo PNAE, criado em meados da década de 1950 com a finalidade de garantir aos alunos a oferta no mínimo de uma refeição diária, durante o seu período de permanência na escola e atualmente propõe-se a suprir parcialmente, no mínimo de 30 a 70\% das necessidades nutricionais dos escolares (Brasil, 2013).

O PNAE também surge como uma possibilidade para o redimensionamento das ações desenvolvidas na escola, podendo ter um papel estratégico para mudanças das práticas alimentares dos escolares, o mesmo tornou-se uma importante estratégia para melhorar a segurança alimentar e nutricional dos alunos através da promoção do direito humano à alimentação adequada (Cunha, 2016).

$\mathrm{Na}$ escola é de extrema importância às interações e atuação do nutricionista e os profissionais da educação, para elucidar a prática da alimentação saudável, a partir do PNAE, com vistas a suprir as necessidades nutricionais diárias, e formação de hábitos alimentares saudáveis, de acordo com a realidade social vivenciada (Almeida, 2014).

É de conhecimento geral que para manutenção do corpo se faz necessário uma alimentação saudável. De acordo Size e Whitney (2013) a alimentação é um dos fatores mais importantes em qualquer fase da vida para promover a saúde e prevenir doenças. Através de uma alimentação balanceada, o organismo obtém energia e nutrientes necessários ao seu desenvolvimento.

De acordo com Almeida (2014), a alimentação oferecida nas escolas é preponderante ao desenvolvimento psicofísico do aluno, auxiliando-o em todos os aspectos: físico motor, intelectual, afetivo emocional, econômico e social. Esses aspectos de bem-estar contribuem para que o sujeito tenha condições satisfatórias para aprender, pois existe um número considerável de estudantes que precisam dessa merenda escolar, para complementar sua refeição principal.

Com isso, percebe-se que a merenda escolar é de vital importância para garantir que as refeições tenham o máximo efeito sobre o estado nutricional, é a única forma de se garantir que os beneficiados, mantenham uma adequada alimentação, garantindo assim que os resultados positivos no estado nutricional e de saúde se mantenham ao longo do tempo. Além disso, a educação em questões de alimentação e nutrição permite que os estudantes desenvolvam conhecimentos ao nível da família, dos amigos e da comunidade (Fonseca, 2015).

Segundo Barros (2012), para que a merenda escolar tenha uma boa aceitação é preciso observar o preparo e a maneira que se serve o prato fazendo uma combinação de cores e texturas para torná-lo atrativo. Há também aspectos que podem facilitar a aceitação da merenda escolar, e um deles é a pesquisa de opinião e hábitos alimentares dos alunos.

Nesse contexto, é citado por Proença (2017), que a alimentação constitui uma das atividades humanas mais importantes, não só por razões biológicas evidentes, mas também por envolver aspectos sociais, psicológicos e econômicos fundamentais na dinâmica da evolução das sociedades. Com relação ao mercado econômico, os recursos gastos com alimentação superam outros setores como o eletrônico e o de armamento. Desta forma, o objetivo geral desse estudo é analisar a importância da merenda escolar para os estudantes.

\section{Metodologia}

Para a realização desse estudo optou-se por uma pesquisa na modalidade de revisão integrativa de literatura. A revisão integrativa determina o conhecimento atual sobre uma temática específica, já que é conduzida de modo a identificar, analisar e sintetizar resultados de estudos independentes sobre o mesmo assunto (Mendes, Silveira \& Galvão, 2008). 
A Revisão Integrativa é um método de pesquisa apontado como ferramenta de grande relevância no campo da saúde, por proporcionar a busca, a avaliação crítica e a síntese de evidências sobre um tema investigado. Esses aspectos facilitam a identificação dos resultados relevantes, de lacunas que direcionam para o desenvolvimento de futuras pesquisas e auxiliam o profissional a escolher condutas e a tomar decisões, proporcionando um saber crítico (Souza, Silva \& Carvalho, 2010).

A coleta das informações para a pesquisa bibliográfica será por meio da exploração da base de dados da Biblioteca Virtual em Saúde (BVS), Biblioteca Cientifica Eletrônica Virtual (SCIELO) e Literatura Latino - Americana e do Caribe em Ciências da Saúde (LILACS).

A busca na base de dados será orientada pelos descritores: "alimentação escolar", "indicadores de saúde comunitária", "alimentos, dieta e nutrição", e será realizado em todos os índices, buscando captar o maior número de artigos publicados no período proposto que abordem a temática em discussão, visando o desenvolvimento fidedigno desse estudo.

Para uma análise crítica e reflexiva dos estudos incluídos na revisão, foi realizada uma leitura minuciosa e criteriosa destacando os que atingirem os critérios de inclusão e que contemplarem o objetivo proposto, para viabilizar o resultado da pesquisa de forma objetiva.

Foram incluídos os artigos publicados em língua portuguesa e inglesa, nos últimos dez anos, com texto completo, disponível on-line, com acesso livre. Foram excluídos artigos que não obedecerem ao tempo de dez anos de publicação, resumos, teses, e trabalhos que não foram publicados em periódicos.

Após as buscas através do cruzamento dos descritores nas bases indexadas: "alimentação escolar", "indicadores de saúde comunitária", "alimentos, dieta e nutrição", foram resultados um total de 849 estudo.

Dos estudos resultantes, um total de 523 foram encontrados na base de dados Scielo, dos quais apenas 245 apresentavam o texto completo disponível, e 67 foram publicados nos últimos cinco anos, resultando após análise crítica um total de 06 artigos que respondem a questão norteadora desse estudo.

Ao que se refere à base de dados Lilacs, após o cruzamento dos descritores foram encontrados um total de 326 estudos, dos quais apenas 197 estudos apresentavam o texto completo e disponível, e apenas 57 estudos foram publicados nos últimos 5 anos obedecendo os critérios previamente estabelecidos, respondendo assim, após uma análise crítica 4 artigo à questão norteadora desse estudo.

Dessa forma, totalizaram um total de 10 estudos que iram compor esse estudo, os quais após análise crítica constatouse que respondem à questão norteadora do estudo aqui proposto, apresentados a partir do corpus desse estudo. 
Quadro 1. Corpus do Estudo.

\begin{tabular}{|c|c|c|c|c|}
\hline N\% ANO & TITULO DO ESTUDO & PERIÓDICO & AUTORES & BASE DE DADOS \\
\hline 2018 & $\begin{array}{l}\text { As ações de educação alimentar e nutricional e o } \\
\text { nutricionista no âmbito do Programa Nacional } \\
\text { de Alimentação Escolar }\end{array}$ & $\begin{array}{l}\text { Ciênc. saúde } \\
\text { colet }\end{array}$ & $\begin{array}{l}\text { SILVA, S. U., MONEGO, } \\
\text { E. T., \& } \\
\text { SOUSA, L. M. }\end{array}$ & SCIELO \\
\hline 2018 & $\begin{array}{l}\text { Avaliação do cardápio e da aceitabilidade da } \\
\text { merenda oferecida em uma escola estadual } \\
\text { de ensino fundamental de Porto Velho, } \\
\text { Rondônia. }\end{array}$ & Saber Científico & $\begin{array}{l}\text { SANTOS, I. H. V. S., } \\
\text { Ximenes, R. M., \& Prado, } \\
\text { D. F. }\end{array}$ & LILACS \\
\hline 2016 & $\begin{array}{l}\text { Serviços de alimentação destinados ao } \\
\text { público escolar: análise da convivência do } \\
\text { Programa de Alimentação Escolar e das } \\
\text { cantinas. }\end{array}$ & $\begin{array}{l}\text { Segurança } \\
\text { Alimentar e } \\
\text { Nutricional }\end{array}$ & $\begin{array}{l}\text { Danelon, M. A. S., } \\
\text { Danelon, M.S., \& Silva, } \\
\text { M.V. }\end{array}$ & LILACS \\
\hline 2016 & $\begin{array}{l}\text { Importância da Alimentação para Melhorias } \\
\text { na Aprendizagem de Crianças em Unidades } \\
\text { Públicas De Ensino. }\end{array}$ & Revista Somma & Lima, A. P. S. C. & SCIELO \\
\hline 2015 & $\begin{array}{l}\text { Exploring nutrition education resources and } \\
\text { barriers, and nutrition knowledge in teachers } \\
\text { in California. }\end{array}$ & $\begin{array}{l}\text { J Nutr Educ } \\
\text { Behav }\end{array}$ & $\begin{array}{l}\text { Jones, A. M., \& } \\
\text { Zidenberg-Cherr, S. }\end{array}$ & SCIELO \\
\hline 2014 & $\begin{array}{l}\text { Organic food-related educational actions } \\
\text { developed by dieticians in Brazilian } \\
\text { municipal schools. }\end{array}$ & Rev Nutr & $\begin{array}{l}\text { Vieira, A. V., Corso, } \\
\text { A.C.T., \& González- } \\
\text { Chica, D.A. }\end{array}$ & SCIELO \\
\hline 2013 & $\begin{array}{l}\text { Alimentação na escola e autonomia - } \\
\text { desafios e possibilidades. }\end{array}$ & $\begin{array}{l}\text { Cien } \quad \text { Saude } \\
\text { Colet }\end{array}$ & $\begin{array}{l}\text { Barbosa, N. V. S., } \\
\text { Machado, N. M. V., } \\
\text { Soares, M.C.V., et al. }\end{array}$ & LILACS \\
\hline 2013 & $\begin{array}{l}\text { Avanços e desdobramentos do marco de } \\
\text { referência da educação alimentar e } \\
\text { nutricional para políticas públicas no âmbito } \\
\text { na universidade e para os aspectos culturais } \\
\text { da alimentação. }\end{array}$ & Rev Nutr & Santos, L.A. & LILACS \\
\hline 2012 & $\begin{array}{l}\text { Perfil do nutricionista do Programa Nacional } \\
\text { de Alimentação Escolar na região Nordeste } \\
\text { do Brasil. }\end{array}$ & Rev Nutr & $\begin{array}{l}\text { Mello, A. L., Vidal Junior, } \\
\text { P. O., \& Sampaio, L. R. }\end{array}$ & SCIELO \\
\hline 2012 & $\begin{array}{l}\text { Abordagem dos temas alimentação e } \\
\text { nutrição no material didático do ensino } \\
\text { fundamental: interface com segurança } \\
\text { alimentar e nutricional e parâmetros } \\
\text { curriculares nacionais. }\end{array}$ & Saúde Soc & $\begin{array}{l}\text { Fiore, E. G., } \\
\text { Jobstraibizer, G. A., } \\
\text { Silva, C.S., et al. }\end{array}$ & SCIELO \\
\hline
\end{tabular}

Fonte: Autores.

\section{Resultados e Discussão}

\subsection{A importância da merenda escolar}

A alimentação constitui uma das atividades humanas mais importantes, não só por razões biológicas evidentes, mas também por envolver aspectos sociais, psicológicos e econômicos fundamentais na dinâmica da evolução das sociedades. Com relação ao mercado econômico, os recursos gastos com alimentação superam outros setores como o eletrônico e o de armamento (Proença, 2017).

Para Cervato-Mancuso et al. (2013), a merenda, além de alimentar e nutrir as crianças, também proporciona interação social entre colegas da escola e as cozinheiras. Os professores estimulam o desenvolvimento de bons hábitos alimentares e ensinam como é o sentar-se à mesa e comer adequadamente. Esses momentos também são importantes para o desenvolvimento afetivo e emocional das crianças.

Na pesquisa realizada por Ribeiro e Silva (2013), percebe-se que a criança deve ter uma alimentação balanceada e controlada na escola e em casa, facilitando ainda mais seu aprendizado, capacidade física, atenção, memória, concentração, 
energia necessária para trabalhar o cérebro. No entanto para que isso se concretize as pesquisas mostram que a alimentação de uma criança deve ser bem diversificada contendo carboidratos, lipídios, proteínas, sais minerais, água, fibras, sal, gorduras, vitaminas na quantidade certa, além da prática de exercícios.

O governo Federal no intuito de oferecer uma merenda escolar de qualidade aos alunos das escolas públicas indica aos órgãos competentes repensarem o cardápio adotado a fim de contribuir para a obtenção de um rendimento escolar mais significativo e ainda manter a saúde (Brasil, 2006). Segundo Lima (2016), a nutrição adequada na infância é importante para o crescimento e desenvolvimento da criança, ao mesmo tempo em que se constitui num dos fatores de prevenção de algumas doenças da idade adulta.

De acordo com Perroni (2013), a importância da merenda escolar e fundamental para o desenvolvimento do estudante na vida escolar e ainda manter uma alimentação nutricional saudável, ou seja, tudo aquilo que ingerimos exerce um grande impacto sobre a função cerebral, podendo interferir no humor, no pensamento, no comportamento, na memória, no aprendizado e no envelhecimento celular.

\subsection{Hábitos alimentares a nível da escola}

A merenda escolar auxilia no desenvolvimento dos hábitos alimentares das crianças e estas, por sua vez, podem se tornar multiplicadoras em suas casas, de modo a incentivar os pais a consumirem alimentos diferentes dos usuais. De acordo com Costa (2016, p. 11), “os hábitos alimentares desenvolvidos durante a infância e adolescência, frequentemente são mantidos na idade adulta".

Com isso, nota-se que os adolescentes seguem um padrão alimentar semelhante aos seus familiares. Estudos demonstram que a preferência por esses lanches, geralmente é com alto conteúdo energético, principalmente proveniente de lipídios, sódio e baixo conteúdo de ferro, cálcio, vitaminas e fibras (Costa, 2016).

A formação dos hábitos alimentares inicia-se logo durante a primeira infância. Isso exige que hábitos saudáveis de alimentação sejam estimulados precocemente. A alimentação saudável é um componente fundamental para a saúde durante todo o curso da vida. Portanto, uma das estratégias fundamentais para a promoção da saúde, da nutrição e para assegurar a promoção da alimentação saudável durante todo o curso da vida (Burity et al., 2010).

Em concordância a isto, Sampaio et al., (2017), afirma que as práticas alimentares de um adulto são formadas por hábitos alimentares adquiridos durante a infância e adolescência e é nesta fase que a interferência e a inclusão dos hábitos alimentares saudáveis são efetivadas. Portanto um ambiente favorável à inclusão de bons hábitos alimentares é a escola, levando está criança ou adolescente a um estilo de vida mais saudável através do conhecimento.

De acordo com o Programa Nacional de Alimentação Escolar (PNAE) busca formar segurança alimentar e nutricional, oferecer hábitos alimentares saudáveis e refeições saudáveis que cubra no mínimo $20 \%$ das necessidades nutricionais de acordo com a faixa etária durante o período de permanência do aluno na escola (Brasil, 2013).

\subsection{Benefícios Nutricionais da Merenda escolar}

$\mathrm{O}$ crescimento do ser humano é dividido em fases, a criança tem um crescimento lento já o adolescente tem um crescimento mais acelerado, devido a essas diferenças o cardápio de uma unidade escolar deve ser elaborado levando em consideração as necessidades de cada fase do desenvolvimento. Uma alimentação pobre e inadequada pode causar doenças nutricionais e psicossociais nesta criança/adolescente (Flávio et al., 2018).

Com isso, percebe-se que para que isto não ocorra é de fundamental importância que os responsáveis em preparar os cardápios e também a merenda escolar tenham conhecimento das necessidades nutricionais dos alunos (Flávio et al., 2018). 
O PNAE, visando limitar a oferta e o consumo de alimentos processados de baixo valor nutricional, ricos em açúcar, gordura e sal estabelece um limite para aquisição de alimentos enlatados, embutidos, doces, alimentos compostos, preparações semiprontas ou prontas para o consumo, ou alimentos concentrados. Proíbe, ainda, a aquisição de bebidas com baixo valor nutricional (Brasil, 2013).

Os benefícios nutricionais provenientes do consumo regular de pescado e o seu baixo consumo reforçam a validade de investimentos e incentivos por meio de políticas públicas para o aumento da disponibilidade e consumo deste alimento no Brasil, assim como a manutenção do padrão de ingestão nas regiões Norte e Nordeste (Santori \& Alan, 2012).

De acordo com Krijstajansson et al., (2007), pesquisas apresentadas em encontro realizado no ano de 2000, a respeito do impacto da alimentação escolar indicaram que existe baixa evidência dos benefícios nutricionais da alimentação escolar, muito embora se possa apresentar fortes indícios de melhorias no processo de aprendizagem. Sobre os benefícios nutricionais que estimula a criatividade para preparar aulas mais interessantes e menos repetitivas para as diferentes faixas etárias.

\section{Conclusão}

Temos o conhecimento que a escola tem um papel importante quanto à orientação da alimentação saudável do seu estudante, pois possui um espaço privilegiado onde poderá desenvolver a promoção da saúde, é importante lembrar que uma alimentação balanceada e equilibrada contendo variedades em alimentos, que tenha tudo que o nosso organismo necessita e a quantidade necessária de água, lipídios, proteínas, vitaminas, sais minerais e carboidratos, pois é através da nossa alimentação de jovens que determinara a nossa saúde do futuro.

As políticas públicas em alimentação e nutrição no Brasil para a promoção da saúde instituiu ações educativas em prol de novos hábitos alimentares e da saúde. O PNAE tem como meta abraçar todo território nacional com suas tendências teóricometodológicas, como a EAN, sendo um programa não somente assistencialista para muitas crianças e adolescente, mas também promotor do direito humano a alimentação adequada.

A merenda escolar oferecida nas escolas pública é importante ao desenvolvimento psicofísico do aluno, auxiliando-o em todos os aspectos: físico motor, intelectual, afetivo emocional, econômico e social. Esses aspectos de bem-estar contribuem para que o sujeito tenha condições satisfatórias para aprender.

Ante o exposto esperamos contribuir para o conhecimento em geral da comunidade escolar e envolvidos sobre a importância da merenda escolar, para o desenvolvimento dos estudantes na vida escolar.

\section{Referências}

Brasil. (2021). Ministério de Educação. Programa de Suplementação: Sobre o PNAE. 2021.

Brasil, Aquisição de Produtos da Agricultura Familiar para o Programa Nacional de Alimentação Escolar Acesso em 15/05/2021 as 13:30 h.

Brasil. (2017). Boas práticas de agricultura familiar para a alimentação escolar/Programa nacional de Alimentação escolar. FNDE.

Brasil. (2015). Ministério da Educação, Cartilha Nacional da Alimentação Escolar, Ministério da Educação, Brasília, DF, 2ª edição.

Brasil. (2013). Ministério da Saúde, Política Nacional de Alimentação e Nutrição Brasília - DF.

Brasil. (2012). Ministério do Desenvolvimento Social e Combate à Fome. Secretaria Nacional de Segurança Alimentar e Nutricional. Marco de Referência de educação alimentar e nutricional para as políticas públicas. Brasília: Ministério do Desenvolvimento Social e Combate à Fome. 68.

Brasil. (2014). Ministério da Educação, Cartilha, Orgânicos na alimentação Escolar a Agricultura Familiar alimentado o saber.

Brasil. (2018). Ministério da Educação, Fundo Nacional de Desenvolvimento da Educação, Manual de apoio para as atividades técnicas do Nutricionista do Âmbito do PNAE, Brasília - DF. 60.

Brasil. Ministério da Educação, Programa Nacional de Alimentação Escolar Cardápios na Alimentação Escolar Coordenação de Segurança Alimentar e Nutricional - COSAN. 
Brasil. Ministério de Educação. Programa de Suplementação: Alimentação e Nutrição < https://www.fnde.gov.br/index.php/programas/pnae/pnae-eixos-deatuacao/pnae-alimentacaoe-nutricao>.

Barbosa, N. V. S., Machado, N. M. V., Soares, M. C. V., \& Pinto, A. R. R. (2013). Alimentação na escola e autonomia - desafios e possibilidades. Cien Saude Colet, 18(4), 937-945.

Bizzo, M. L. G., \& Leder, L. (2005). Educação nutricional nos parâmetros curriculares nacionais para o ensino fundamental. Rev Nutr. 18(5), 661-667.

Burity, V., Franceschini, T., Valente, F., Recine, E., Leão, M., \& Carvalho, M. F. (2010). Direito Humano à Alimentação Adequada no Contexto da Segurança Alimentar e Nutricional. Brasília.

Caniné, E. S., \& Ribeiro, V. M. B. (2007). A prática do nutricionista em escolas municipais do Rio de Janeiro: um espaço tempo-educativo. Ciênc Educ. 13(1), 47-70.

Camozzi, A. B. et al. (2015). O Promoção da Alimentação Saudável na Escola: realidade ou utopia? Cad. Saúde Colet., Rio de Janeiro, 23 (1), $32-7$.

Cervato-Mancuso, A. M., Westphal, M. F., Arak, E. L., \& Bógus, C. M. (2013). O Papel da Alimentação escolar na formação dos hábitos alimentares. Ver. Paul. Pediatr., São Paulo, 31(3), 324- 330.

Costa, E. Q., Ribeiro, V. B., \& Ribeiro, E. C. O. (2011). Programa de alimentação escolar: espaço de aprendizagem e produção de conhecimento. Rev Nutr. 14(3), 225-229.

Danelon, M. A. S., Danelon, M. S., \& Silva, M. V. (2016). Serviços de alimentação destinados ao público escolar: análise da convivência do Programa de Alimentação Escolar e das cantinas. Segurança Alimentar e Nutricional. 13: Unicamp, 85-94.

Davanço, G. M., Taddei, J. A. A. C., Gaglianone, C. P. (2004). Conhecimentos, atitudes e práticas de professores de ciclo básico, expostos e não expostos a curso de educação nutricional. Rev Nutr, 17(2), 177-184.

Fiore, E. G., Jobstraibizer, G. A., Silva, C. S., \& Cervato-Mancuso, A. M. (2012). Abordagem dos temas alimentação e nutrição no material didático do ensino fundamental: interface com segurança alimentar e nutricional e parâmetros curriculares nacionais. Saúde, 21(4), 1063-1074.

Fonseca, A. N., \& Gomes, J. C. (2015). Merenda Escolar: um Estudo Exploratório sobre a Implementação do Programa Nacional Alimentação na Escola PNAE, na Unidade Integrada Padre Newton Pereira em São Luís, EDUCARE, PUC.

FNDE, Agencia Nacional de Vigilância Sanitária. (2013). Guia de Instruções das Ferramentas para as Boas Práticas na Alimentação Escolar, Brasília.

Flávio, E. F., Barcelos, M. F. P., \& Lima, A. L. (2004). Avaliação química e aceitação da merenda escolar de uma escola estadual de Lavras-MG. Cienc agrotec, 28(4):840-847

Guia Prático. (2020). Alimentação escolar indígena e de comunidades tradicionais, PNAE Indígena no Amazonas, Brasília.

Hernandez, A. B., Slavutzky, S. M. B., \& Padilha, D. M. P. (2008). Avaliação do consumo da merenda escolar em escolas municipais de Porto Alegre. Rev Fac Odonto. Porto Alegre. 49(1), 26-30.

Jesus, A. P. (2016). Alimentação Saudável na Escola: Promovendo A Saúde e Construindo Ações de Segurança Alimentar, TCC.

Jones, A. M., \& Zidenberg-Cherr, S. (2015). Exploring nutrition education resources and barriers, and nutrition knowledge in teachers in California. $J$ Nutr Educ Behav. 47(2), 162-169.

Lima, Ana Patrícia Silva Carvalho. (2016). Importância da Alimentação para Melhorias na Aprendizagem de Crianças em Unidades Públicas De Ensino. Revista Somma, Teresina, 2(2), 74-83.

Marlette, M. A., Templeton, S. B., \& Panemangalore, M. (2005). Food Type, Food Preparation, and Competitive Food Purchases Impact School Lunch Plate Waste by Sixth-Grade Students. J Am Diet Assoc, 105(11), 1779-1782.

Marconi, M. A., \& Lakatos, E. M. (2010). Fundamentos de metodologia científica. Editora Atlas.

Mello, A. L., Vidal Junior, P. O., Sampaio, L. R., Santos, L. A. S, Freitas, M. C. S, \& Fontes, G. A. V. (2012). Perfil do nutricionista do Programa Nacional de Alimentação Escolar na região Nordeste do Brasil. Rev Nutr, 25(1), 119-132.

Mendes, K. D. S., Silveira, R. C. C. P., \& Galvão, C. M. (2008). Revisão integrativa: método de pesquisa para a incorporação de evidências na saúde e na enfermagem. Texto Contexto Enferm. 17(4), 758-64.

Morais, A K., Lima, U. M. F., Nobrega Neta, M. F., Araújo, L. S., Nobrega, L. M., \& Nobrega, M. M. (2018). O Papel Da Agricultura Familiar No Programa Nacional De Alimentação Escolar: Uma Revisão Literária, João Pessoa.

Muller. (2011). Boas Práticas de Manipulação de Alimentos com Merendeiras. São Miguel do Oeste, Uniedu.

Muniz, V. M., \& Carvalho, A. T. (2017). O Programa Nacional de Alimentação Escolar em município do estado da Paraíba: um estudo sob o olhar dos beneficiários do Programa. Revista de Nutrição, 20(3) Campinas.

Neto, L. G., Bezerra, J. A. B., \& Santos, A. N. (2012). Qualidade na merenda escolar: intervenções gastronômicas. Encontro Nacional de Didática e Práticas de Ensino Campinas: UNICAMP.

Oliveira, Rosimaire Valente. (2016). O Programa Nacional De Alimentação Escolar: Uma Análise De Sua Implementação Nas Escolas Da Rede Estadual De Ensino De Manaus, Juiz De Fora. 
Perroni, Cristiane. (2013). Boa alimentação interfere na função cerebral e aumenta a concentração.

Proença, R. P. C. (2017). Alimentação e globalização: algumas reflexões, Cienc. Cult. 62(4).

Ribeiro, G. N. M., \& Silva, J. B. L. (2013). Alimentação no Processo de aprendizagem. Revista Eventos Pedagógicos, Mato Grosso, 4(2), 77-85.

Ribeiro, Ana L.P., Silene C., Djulia T.B. (2013). Programa Nacional de Alimentação Escolar (PNAE) e a participação da agricultura familiar em municípios do Rio Grande do Sul, Revista Gestão e Desenvolvimento em Contexto- Gedecon, 1(01), 36-49.

Rodrigues, L. P. F., Zaneti, I. C.B., \& Laranjeira, N. P. (2012). Sustentabilidade, segurança alimentar e gestão ambiental para a promoção da Saúde e qualidade de vida.

Schneider, S. (2003). Teoria social, agricultura familiar e pluriatividade. Revista Brasileira de Ciências Sociais, 18(51), 99-121.

Santos, L. A. S. (2012). O fazer educação alimentar e nutricional: algumas contribuições para reflexão. Cien Saude Colet. 17(2), 453-462.

SEDUC, Secretaria de Educação do Estado do Amazonas. Programa de Regionalização da Merenda Escolar (Preme), < http://www.educacao.am.gov.br/programa-de-regionalizacao-damerenda-escolar-preme/>.

Santos, I. H. V. S., Ximenes, R. M., \& Prado, D. F. (2008). Avaliação do cardápio e da aceitabilidade da merenda oferecida em uma escola estadual de ensino fundamental de Porto Velho, Rondônia. Saber Científico, Rondônia, 1(2), 100-111. http://revista.saolucas.edu.br/index.php/resc/article/view/24/ED26.

Santos, Deborah Maria. (2017). TCC - a Alimentação Escolar como Estratégia de Educação Alimentar e Nutricional: Uma Revisão da Literatura, Vitória de Santo Antão.

Santori, A., \& Amancio, R. D. (2012). Pescado: importância nutricional e consumo no Brasil. Segurança Alimentar e Nutricional, Campinas, $19(2)$, 83-93.

Sampaio, H. A. C., Sabry, M. O. D., Rêgo, J. M. C., Passamai, M. P. B., Sá, M. L. B., Matos, M. R. T., \& Passos, T. U. (2017). Estado nutricional de escolares de um bairro da periferia da cidade de Fortaleza - Ceará. Revista Nutrição em Pauta, 84.

Souza, M. T., Silva, M. D., \& Carvalho, R. (2010). Revisão integrativa: o que é e como fazer. Einstein. 8(1 Pt 1), $102-6$.

Vieira, A. V., Corso, A. C. T., \& González-Chica, D. A. (2014). Organic food-related educational actions developed by dieticians in Brazilian municipal schools. Rev Nutr. 27(5):525-535.

Watts, S. O., Piñero, D. J, Alter, M. M., Lancaster, K. J. (2012). An Assessment of nutrition education in selected counties in New York State elementary schools. J Nutr Educ Behav. 44(6):715-725. 